\title{
Propiedades psicométricas del Inventario de Ansiedad de Beck en adultos asmáticos mexicanos ${ }^{1}$
}

\section{Psychometric properties of the Beck Anxiety Inventory in Mexican asthmatic adults}

\author{
Centli Guillén Díaz-Barriga y Ana Luisa González-Celis Rangel²
}

Citación: Guillén D.B., C. y González C.R., A.L. (2019). Propiedades psicométricas del Inventario de Ansiedad de Beck en adultos asmáticos mexicanos. Psicología y Salud, 29(1), 5-16.

RESUMEN

\begin{abstract}
Los individuos con asma suelen exhibir un elevado índice de sintomatología ansiosa que, a su vez, se asocia con un control deficiente de la enfermedad. Por lo anterior, se requiere una herramienta práctica que identifique a pacientes con tales síntomas para así brindarles una atención psicológica oportuna. El Inventario de Ansiedad de Beck es uno de los instrumentos más utilizados en población general y con enfermedades crónicas; sin embargo, no se han estudiado sus características en población asmática. Así, el objetivo del presente estudio fue determinar las propiedades psicométricas y estructura factorial de dicho instrumento en adultos asmáticos mexicanos, para lo cual se empleó un diseño ex post facto transversal con un muestreo no probabilístico por conveniencia. Participaron 157 asmáticos usuarios de un instituto de salud pública de la Ciudad de México que aguardaban consulta médica. Los instrumentos utilizados fueron el Inventario de Ansiedad de Beck, el Inventario de Depresión de Beck y el Cuestionario de Control del Asma. El análisis factorial exploratorio inicial produjo cuatro factores y excluyó dos reactivos. Los 19 reactivos restantes se agruparon en cuatro factores y explicaron $59.93 \%$ de la varianza. El factor denominado "síntomas de respiración" agrupó síntomas de ansiedad similares a los asmáticos. El valor del coeficiente alfa de Cronbach fue satisfactorio y se obtuvieron valores de correlación moderados con el Inventario de Depresión de Beck y el Cuestionario de Control del Asma. Se concluye que el Inventario de Depresión de Beck es válido y confiable para emplearse población asmática mexicana.
\end{abstract}

Palabras clave: BAI; Ansiedad; Asma; Análisis factorial exploratorio; Propiedades psicométricas.

\begin{abstract}
Individuals with asthma show high rates of anxious symptomatology, which is in turn associated with poor asthma control. Therefore, a practical tool that identifies patients with anxiety symptoms is required to provide timely psychological care. The Beck Anxiety Inventory is one of the most used instruments in general population and with chronic diseases; however, its characteristics among asthmatic population have not been studied. The aim of this study was to determine the psychometric properties and factor composition of the BAI on a sample of Mexican asthmatic adults. A descriptive, ex post facto transversal design was used with a non-probabilistic convenience sampling. Participants included 157 asthmatic outpatients of a public health institute in Mexico City. The evaluation was carried out in the waiting room while patients waited for medical consultation. The instruments answered by the patient were the Beck Anxiety Inventory, the Beck Depression Inventory, and the Asthma Control Questionnaire. The initial exploratory factor analysis produced
\end{abstract}

\footnotetext{
${ }^{1}$ El presente artículo forma parte de la tesis doctoral de la primera autora bajo la dirección de la segunda.

${ }^{2}$ Facultad de Estudios Superiores Iztacala, Universidad Nacional Autónoma de México, Av. De los Barrios 1, Los Reyes Iztacala, 54090 Tlalnepantla, Edo. de México, México, tel. (55)27-01-97-74, correos electrónicos: centlig@hotmail.com y algcr10@hotmail.com. Artículo recibido el 15 de junio y aceptado el 31 de agosto de 2017.
} 
four factors with two deleted items. The remaining 19 items were grouped into four factors and explained $59.93 \%$ of the variance. The factor "breathing symptoms" grouped the symptoms of anxiety which share similarities with the signs of asthmatic crises. The Cronbach alpha value was satisfactory, and moderate correlation values were obtained with the Beck Depression Inventory and the Asthma Control Questionnaire. It is concluded that the Beck Anxiety Inventory is a valid and reliable instrument to be used in the Mexican asthmatic population.

Key words: BAI; Anxiety; Asthma; Factorial exploratory analysis; Psychometric properties

\section{INTRODUCCIÓN}

$\mathrm{E}$ n diversos estudios realizados en el mundo se ha reportado que los adultos que padecen asma bronquial tienen hasta seis veces más probabilidades de exhibir un trastorno de ansiedad que la población general (Goodwin, Jacobi y Thefeld, 2003). En algunas investigaciones se ha reportado que entre $36.9 \%$ (Ciprandi, Schiavetti, Rindone y Ricciardolo, 2015) y 47.3\% (Cooper et al., 2007) de la población asmática presentan o presentarán dichos síntomas en algún momento de la enfermedad. Incluso se ha reportado que tales pacientes tienen niveles de ansiedad más altos que aquellos con otras enfermedades crónicas, como EPOC (Braket et al., 2015). En el caso de México, Lugo, Reynoso y Fernández (2014) identificaron que $39.6 \%$ de los pacientes con asma padecen síntomas de ansiedad.

Lo anterior es relevante porque se ha encontrado que la ansiedad tiene diversas consecuencias perjudiciales, como un deterioro significativo del control de la enfermedad (Lavoie, Bodreau, Plourde, Campbell y Bacon, 2011), un mayor riesgo de exacerbaciones asmáticas (Liu et al., 2014) y una mayor frecuencia de visitas a la sala de urgencias y hospitalizaciones (Tany y Saha, 2017). El paciente ansioso percibe con más intensidad los síntomas del asma (Li et al., 2015); es más propenso a sufrir otros problemas psicológicos, como la depresión (Brumpton et al., 2013); muestra una escasa adhesión terapéutica al tratamiento médico (Ritz, Meuret, Trueba, Fritzsche y von Leupoldt, 2013) $y$ en general experimenta un deterioro de su nivel de calidad de vida relacionada con la enfermedad
(Lomper, Chudiak, Uchmanowicz, Rosinczuk y Jankowska-Polanska, 2016) y de su calidad de vida en general (Coban y Aydemir, 2014; Correia et al., 2013). Por consiguiente, la valoración de la ansiedad en pacientes asmáticos se vuelve necesaria, por lo que es indispensable contar con un instrumento sensible que aporte mediciones válidas y confiables y que garantice la detección certera y oportuna de los síntomas de ansiedad en esta población, a fin de no confundir la sintomatología ansiosa con otros síntomas asociados a la enfermedad.

Entre la amplia gama de instrumentos que se han diseñado para evaluar la sintomatología de ansiedad, el Inventario de Ansiedad de Beck (Beck, Epstein, Brown y Steer, 1988), conocido como BAI por sus siglas en inglés, es una de las herramientas que se emplean con mayor frecuencia en todo el mundo, tanto en el ámbito de la investigación psicológica como en el campo clínico (Bardhoshi, Duncan y Erford, 2016). Si bien no es un instrumento que tenga como fin diagnosticar los trastornos de ansiedad, pues no los clasifica y solamente indica la severidad de la sintomatología, su popularidad radica en su aplicación sencilla e interpretación práctica, y en que provee datos valiosos para una primera aproximación a dichos trastornos (Sanz, 2014).

El BAI surgió como una propuesta para valorar la gravedad de los síntomas de ansiedad de una persona y discriminar entre síntomas de ansiedad y de depresión. En su evaluación original, Beck et al. (1988) obtuvieron una consistencia interna alta $(\alpha=.92)$, una confiabilidad test-retest de .75 , y además una correlación moderada con la Escala de Ansiedad de Hamilton (Hamilton. 1969) $(\mathrm{r}=.51)$, y baja con la Escala de Depresión del mismo autor (Hamilton, 1967) $(r=.25)$. La estructura factorial obtenida fue de dos dimensiones: una compuesta por síntomas somáticos (doce reactivos) y la otra por los síntomas subjetivos de la ansiedad relacionadas con el trastorno de pánico (nueve reactivos).

Posteriormente, Beck y Steer (1991) llevaron a cabo una validación en 367 participantes con diagnósticos de trastornos de ansiedad y del estado del ánimo. La aportación principal de ese estudio fue que el análisis factorial arrojó cuatro factores: síntomas subjetivos, neurofisiológicos, autonómicos y característicos de ataques de pánico. Desde la 
publicación del mismo a la fecha se han generado diversas investigaciones en población general y psiquiátrica para determinar las propiedades del BAI. Por tal motivo, Bardhoshi et al. (2016) 1levaron a cabo un exhaustivo metaanálisis de sus propiedades psicométricas mediante la recolección de los estudios comprendidos entre 1993 y 2013. Los resultados obtenidos proporcionan estimaciones de consistencia interna fuerte tanto en población clínica $(\alpha=.91, \mathrm{k}=61, \mathrm{n}=18,015)$ como en población general $(\alpha=.91, \mathrm{k}=56, \mathrm{n}=25,917)$. En lo referente a la composición factorial, nueve estudios de los dieciocho reportados eran similares al estudio original y arrojaron dos dimensiones: síntomas somáticos y síntomas subjetivos (Contreras, Fernández, Malcame, Ingram y Vaccarino, 2004; Kabacoff, Segal, Hersen y Van Hasselt, 1997; Kumar, Steer y Beck, 1993; Lovibond y Lovibond, 1995; Peterson, 1995; Sanford, Bush, Stone, Lichstein y Aguillard, 2008; Steer, 2009; Steer, Kumar, Ranieri y Beck, 1995; Steer, Rissmi1ler, Ranieri y Beck, 1993); cuatro estudios propusieron un modelo de cuatro factores: subjetivo, neurofisiológico, autónomo y pánico (Durham, 2010; Osman, Barrios, Aukes, Osman y Markway, 1993; Steer, Kumar y Beck, 1993; Osman et al., 2002). Un estudio más propuso un modelo de cinco factores: somático, subjetivo, síntomas somáticos relacionados con la temperatura, síntomas físicos de asfixia, y pánico (Lindsay y Skene, 2007), y finalmente otro propuso seis factores: somático, miedo, hiperactividad autonómica, pánico, nerviosismo y tensión motora (Morin et al., 1999).

En el caso específico de la población mexicana, Robles, Varela, Jurado y Páez (2001) obtuvieron una estructura de cuatro factores: síntomas subjetivos, neurofisiológicos, autonómicos y de pánico. Esta versión en español mostró una alta consistencia interna, medida por el coeficiente alfa de Cronbach, de .84 en estudiantes universitarios y de .83 en adultos de población general. Al comparar población general y pacientes con trastorno de ansiedad, se reportaron diferencias significativas entre las puntuaciones promedio de la escala $(t=19.11$, $p<.05)$, lo que indica su poder de discriminación.

Por otra parte, la estructura factorial del BAI también ha generado interés en lo que atañe a la población con enfermedades fisiológicas, debido principalmente a la posible superposición que hay entre los síntomas somáticos asociados con el diagnóstico médico y los síntomas somáticos que reflejan ansiedad (Clark et al., 2016; Kagee, Coetzee, Saal y Nel, 2015; Phan et al., 2016). En la investigación realizada por Clark et al. (2016) se hace énfasis en que los pacientes que sufrieron un evento cardiaco manifiestan una estructura similar en los procesos de pensamiento que los de la población general o psiquiátricos; empero, los síntomas somáticos se agrupan de forma diferente debido a las experiencias orgánicas debidas a la enfermedad que han tenido los pacientes.

En el caso específico de la población asmática mexicana, el BAI ha sido el instrumento de elección de los investigadores. Se ha empleado tanto para obtener las cifras de prevalencia de la ansiedad (Lugo et al., 2014), como para evaluar los efectos de una intervención cognitivo-conductual dirigida a la adherencia terapéutica y el tratamiento de la ansiedad (Caballero, Fernández, Salas, Chapela y Sánchez-Sosa, 2009). Sin embargo, no se cuenta con datos de la estructura factorial del instrumento en dicha población.

Debe apuntarse que Robles et al. (2001) señalan que el BAI tiene propiedades psicométricas idóneas para ser utilizado en población mexicana con trastornos psiquiátricos y población general, pero recomiendan que se evalúe la pertinencia del BAI estandarizado en otras muestras.

A pesar de que la interpretación del BAI se hace con base en el resultado total y no por sus factores, conocer las dimensiones que subyacen al instrumento puede proporcionar información para generar estrategias de tratamiento específicas a la población de estudio, sobre todo considerando la gran variabilidad de la composición factorial en otras muestras de pacientes crónicos, y que los síntomas característicos del asma (dificultad para respirar, falta de aire, sensación de asfixia, opresión torácica y palpitaciones cardiacas) (Iniciativa Global para el Asma [GINA], 2015) también son síntomas fisiológicos que aparecen en la ansiedad.

De ahí que el objetivo del presente estudio no experimental, descriptivo-correlacional, con diseño ex post facto transversal, haya sido determinar las características psicométricas y la estructura factorial del Inventario de Ansiedad de Beck (BAI) en una muestra de pacientes adultos con diagnóstico de asma bronquial. 


\section{MÉTODO}

\section{Participantes}

Los participantes fueron reclutados a lo largo de tres meses en la clínica de asma de un instituto de salud pública de tercer nivel de atención ubicado en la Ciudad de México.

Los criterios de inclusión fueron tener una edad igual o mayor a 17 años y un diagnóstico de asma confirmado por el neumólogo tratante del instituto, sin importar el grado de severidad de la enfermedad. A su vez, los de exclusión fueron dejar inconcluso $15 \%$ o más de algún instrumento de evaluación o retirarse voluntariamente de la investigación.

\section{Muestreo}

Se empleó un muestreo no probabilístico por conveniencia. Se estableció el tamaño de la muestra teniendo en cuenta que para poder llevar a cabo los procedimientos factoriales se necesitan al menos cinco participantes por cada reactivo del instrumento que se somete al análisis (Carretero y Pérez, 2005). En virtud de que el BAI se compone de 21 reactivos, la cifra mínima de participantes se estableció en 105. En el estudio se alcanzó un tamaño muestral de 157 pacientes adultos asmáticos.

\section{Instrumentos}

Ficha de identificación. Contiene información de algunas características sociodemográficas de los pacientes, esto es, sexo, edad, estado civil y escolaridad.

Inventario de Ansiedad de Beck (BAI) (Beck et al., 1988). La variable de ansiedad se evaluó mediante este instrumento, conformado por 21 reactivos. Al aplicarse, se pide a los respondientes que indiquen cuánto han sido afectados por una serie de síntomas durante la última semana. La calificación se hace a través de una escala tipo Likert de 0 a 3, donde 0 significa la ausencia del síntoma y $3 \mathrm{su}$ severidad máxima. El puntaje total se obtiene de la suma de cada uno de los reactivos, siendo 0 el puntaje mínimo y 63 el máximo. Las normas de calificación en población mexicana son, a saber: de 0 a 5 puntos, ansiedad mínima; de 6 a 15, ansiedad leve; de 16 a 30 puntos, ansiedad moderada, $y$ de 31 a 63, ansiedad severa. El criterio para considerar la ansiedad como clínicamente relevante es obtener 16 puntos o más. La versión mexicana para adultos se caracteriza por una alta consistencia interna $(\alpha=.83)$, elevado coeficiente de confiabilidad test-retest $(\mathrm{r}=.75)$ y una estructura factorial de cuatro componentes principales (Robles et al., 2001).

Inventario de Depresión de Beck (BDI) (Beck, Steer y Brown, 1996). Instrumento autoadministrable de 21 reactivos con los que se evalúa la gravedad de la sintomatología depresiva. Cada reactivo se califica de 0 a 3 . La suma de todas sus respuestas varía de 0 a 63 puntos. Cuanto mayor es el resultado obtenido, mayor es la severidad de los síntomas depresivos. Un resultado superior a 10 revela presencia de depresión. Este instrumento está traducido, adaptado y estandarizado por Jurado et al. (1998) para población mexicana, con una validez concurrente adecuada $(\mathrm{r}=.70)$ y alta consistencia interna $(\alpha=.87)$. El índice de confiabilidad del cuestionario obtenido en la presente investigación fue de .88 .

Cuestionario del Control del Asma (ACT) (Nathan et al., 2004). La función de este instrumento fue medir el control de la enfermedad asmática. Se encuentra conformado por cinco preguntas que evalúan la frecuencia con que los síntomas asmáticos han interferido con las actividades diarias durante el último mes. Cada pregunta cuenta con cinco opciones de respuestas tipo Likert que se califican de 1 a 5 puntos; un mayor puntaje indica un mejor control del asma, y puntajes menores a 19 indican que el asma no está controlada. Es utilizado y recomendado por neumólogos mexicanos para evaluar el control de los síntomas asmáticos (Larenas et al., 2017). El índice de confiabilidad del cuestionario obtenido en la presente investigación fue de .77 .

\section{Procedimiento}

El reclutamiento de los pacientes se realizó en las instalaciones de la clínica de asma del instituto. Todos los pacientes que aguardaban en la sala de 
espera de la clínica de asma para entrar a consulta médica eran candidatos a participar. Únicamente debían confirmar los criterios de inclusión, para lo cual se les preguntó su edad y si contaban con el diagnóstico de asma confirmado por su médico tratante.

Luego, el investigador explicó de manera sencilla los objetivos de la investigación e invitó a los pacientes a formar parte de la misma. Se les informó que los instrumentos debían ser completados en una única sesión y mientras esperaban la consulta con el neumólogo tratante. La batería de instrumentos fue llenada de forma individual. Con aquellos participantes que no supieran leer, escribir o tuvieran impedimentos visuales, el investigador aplicó los instrumentos en forma de entrevista. El tiempo para llenar los instrumentos varió entre 15 y 30 minutos por participante. Todos ellos colaboraron de manera voluntaria, confidencial, consentida e informada.

\section{Análisis de datos}

Los datos se procesaron con el programa estadístico SPSS, versión 20.0. En las variables categóricas (sexo, estado civil y escolaridad), se calcularon sus frecuencias y porcentajes, mientras que en las variables continuas (edad, ansiedad, depresión y control de la enfermedad) se obtuvieron medidas de tendencia central: media y desviación estándar.

Para el análisis factorial exploratorio se recurrió al método de componentes principales con rotación varimax ortogonal. Los criterios para considerar un factor fue que tres o más reactivos con cargas mayores a .40 se agruparan y que hubiera congruencia teórica entre ellos (Reyes y García, 2008), y para obtener el índice de consistencia interna del instrumento se calculó el coeficiente alfa de Cronbach.

Para calcular la validez convergente del BAI, se computó el coeficiente de correlación de Pearson con las medidas totales los instrumentos BDI y ACT. De igual manera, se calculó el coeficiente de Pearson de las preguntas del BAI que hacen alusión a síntomas respiratorios (reactivos 7, 11 y 15) con el reactivo de la escala ACT que pregunta específicamente al paciente por la sensación de falta de aire debida al asma (reactivo 2). Para este análisis, se invirtió el sentido original del reactivo del ACT, indicando así que a menor puntaje, mejor control de la enfermedad.

Asimismo, se compararon los puntajes totales de mujeres y hombres por medio de una prueba $t$ de Student para grupos independientes.

Luego del análisis factorial, y con el propósito de adecuar los puntos de corte a los puntajes mínimos y máximos obtenidos en el estudio, así como determinar los puntos de corte para los distintos niveles de ansiedad, se empleó la fórmula de relación de linealidad

Valor calculado $=(x * 100 / n)$, donde $x$ representa la puntuación que se va a transformar y $n$ es igual a la puntuación máxima total de la escala.

Los puntajes que se emplearon para comparar y determinar los puntos de corte para los distintos niveles de ansiedad fueron los obtenidos por Robles et al. (2001) en población mexicana, que también fueron convertidos mediante la relación de linealidad. Finalmente, se obtuvieron los valores percentilares para mujeres, varones y muestra total.

\section{RESULTADOS}

En la Tabla 1 se muestran las características sociodemográficas de la muestra. Participaron un total de 157 asmáticos de entre 17 y 68 años de edad $(\mathrm{M}=40.98$, D.E. $=13.97)$. La mayoría se encontraba casado $(52.9 \%)$ y contaba con estudios de educación básica (64.4\%). El promedio de puntuación del BAI fue de 11.45 y D.E. de 9.35; el BDI obtuvo una media de 11.03 y D.E. de 9.95 , y el ACT una media de 17.31 y D.E. de 4.13.

El índice de adecuación Kayser-Meyer-Olkin (KMO) fue de .86, por lo que se llevó a cabo el análisis factorial. El análisis factorial exploratorio inicial arrojó cuatro factores (diecinueve reactivos) y un indicador (dos reactivos) que explican $62.05 \%$ de la varianza (Tabla 2). 
Tabla 1. Características sociodemográficas de los participantes.

\begin{tabular}{|c|l|r|l|l|r|}
\hline & Categoría & \multicolumn{1}{|c|}{ n (\%) } & \multicolumn{2}{|c|}{ Categoría } & \multicolumn{1}{|c|}{ n (\%) } \\
\hline \multirow{5}{*}{ Sexo } & & & Soltero & $56(35.7)$ \\
& & & & Casado & $83(52.9)$ \\
& Mujeres & $112(71.3)$ & \multirow{4}{*}{ Estado civil } & Divorciado & $10(6.4)$ \\
& Hombres & $45(28.7)$ & & Viudo & $5(3.2)$ \\
& & & & No especifica & $3(1.9)$ \\
\hline \multirow{5}{*}{ Edad } & 17 a 29 & $37(23.6)$ & & Analfabetos & $5(3.2)$ \\
& 30 a 39 & $37(23.6)$ & & Educación básica & $101(64.4)$ \\
& 40 a 49 & $43(27.4)$ & Escolaridad & Licenciatura & $31(19.7)$ \\
& 50 a 59 & $23(14.6)$ & & Posgrado & $6(3.8)$ \\
& 60 a 69 & $13(8.3)$ & & No especifica & $14(8.9)$ \\
& 70 a 78 & $4(2.5)$ & & & \\
\hline
\end{tabular}

Tabla 2. Matriz factorial rotada del Inventario de Ansiedad de Beck para asmáticos mexicanos.

\begin{tabular}{|l|r|r|r|r|r|}
\hline \multirow{2}{*}{ Reactivos } & \multicolumn{4}{|c|}{ Factores } \\
\cline { 2 - 5 } & \multicolumn{1}{|c|}{$\mathbf{1}$} & $\mathbf{2}$ & $\mathbf{3}$ & \multicolumn{1}{|c|}{$\mathbf{5}$} & $\mathbf{5}$ \\
\hline 9. Terror & .807 & & & & \\
17. Asustado & .717 & & & & \\
8. Inseguridad & .650 & & & & \\
14. Miedo a perder el control & .574 & & & & \\
10. Nerviosismo & .561 & & & & \\
16. Miedo a morir & .551 & & & & \\
4. Dificultad para relajarse & .475 & & & & \\
5. Miedo a que pase lo peor & .406 & & & & \\
\hline 13. Cuerpo tembloroso & & .743 & & & \\
3. Debilitamiento de las piernas & & .727 & & & \\
19. Debilidad & & .611 & & & \\
12. Manos temblorosas & & .460 & & & \\
6. Sensación de mareo & & & .864 & & \\
\hline 15. Dificultad para respirar & & & .684 & & \\
11. Sensación de ahogo & & & & .770 & \\
7. Opresión en el pecho o latidos acelerados & & & .662 & & \\
\hline 2. Oleadas de calor (bochornos) & & & & .767 & \\
20. Ruborizarse, sonrojamiento & & & & .748 & \\
21. Sudoración (no debida a calor) & & & & & .651 \\
\hline 18. Indigestión o malestar estomacal & & & & & .641 \\
1. Entumecimiento & $\mathbf{3 4 . 1 9}$ & $\mathbf{8 . 9 0}$ & $\mathbf{7 . 1 9}$ & $\mathbf{6 . 4 6}$ & $\mathbf{5 . 2 9 0}$ \\
\hline \% Varianza explicada & $\mathbf{3 4 . 1 9}$ & $\mathbf{4 3 . 1 0}$ & $\mathbf{5 0 . 2 9}$ & $\mathbf{5 6 . 7 6}$ & $\mathbf{6 2 . 0 5 0}$ \\
\% Varianza acumulada & $\mathbf{. 7 5 8}$ & $\mathbf{. 7 5 5}$ & $\mathbf{. 7 8 3}$ & $\mathbf{. 7 5 7}$ & $\mathbf{. 4 5 6}$ \\
\hline Alfa de Cronbach & & & & & \\
\hline
\end{tabular}

Nota: Método de extracción de análisis de componentes principales. Método de rotación: Varimax con normalización Kaiser. La rotación convergió en siete iteraciones.

El primer factor agrupó ocho reactivos, los cuales hacen alusión a los síntomas subjetivos de la ansiedad, con $34.19 \%$ de la varianza explicada y un valor $\alpha$ de .85; el segundo factor, conformado por cinco reactivos, agrupó los síntomas neurológicos con una varianza explicada de $8.9 \%$ y un valor $\alpha$ de .75; el tercer factor, compuesto por tres reactivos que agrupaban síntomas respiratorios y que obtuvieron un valor $\alpha$ de .78, explicó $7.78 \%$ de la varianza; el cuarto factor, respuestas autonómicas, se compuso de tres reactivos y obtuvo un valor $\alpha$ de .757 , con $6.94 \%$ de la varianza explicada. Finalmente, para un indicador compuesto de dos reactivos (reactivos 1 y 18), con $\alpha$ de .456, una varianza explicada de $5.29 \%$ y un índice de confiabilidad $\alpha$ de .89 . Se tomó la decisión de excluir 
los reactivos 1 y 18 debido a que no cumplían con los requisitos previamente establecidos para ser considerados un factor (esto es, que se agrupen tres o más reactivos con cargas mayores a $.40 \mathrm{y}$ que haya congruencia teórica entre ellos) y a que la confiabilidad del instrumento no se veía comprometida con tal eliminación. Por lo tanto, se llevó a cabo un segundo análisis exploratorio sin los mencionados reactivos.

En este segundo análisis exploratorio, el índice KMO obtenido fue de .89. Como puede observarse en la Tabla 3 , se conservaron los cuatro factores que explican $59.9 \%$ de la varianza acumulada: el primer factor, síntomas subjetivos (seis reactivos), con $35.78 \%$ de la varianza explicada $\mathrm{y}$ un índice $\alpha$ de .85; el segundo factor, síntomas neurofisiológicos (seis reactivos), con $9.42 \%$ de varianza explicada y un índice $\alpha$ de .75; el tercer factor, síntomas de respiración (tres reactivos), con $7.78 \%$ de varianza explicada y un índice $\alpha$ de .78 , y finalmente el cuarto factor, síntomas autonómicos (tres reactivos), con $6.9 \%$ de varianza acumulada y un índice $\alpha$ de .75 .

Tabla 3. Matriz factorial rotada del Inventario de Ansiedad de Beck para asmáticos mexicanos sin reactivos eliminados.

\begin{tabular}{|c|c|c|c|c|c|}
\hline \multirow{2}{*}{ Factores } & \multirow{2}{*}{ Reactivos } & \multicolumn{4}{|c|}{ Factores } \\
\hline & & 1 & 2 & 3 & 4 \\
\hline \multirow{6}{*}{ 1. Síntomas subjetivos } & 9. Terror & .799 & & & \\
\hline & 8. Inseguridad & .704 & & & \\
\hline & 17. Asustado & .668 & & & \\
\hline & 14. Miedo a perder el control & .598 & & & \\
\hline & 16. Miedo a morir & .545 & & & \\
\hline & 5. Miedo a que pase lo peor & .545 & & & \\
\hline \multirow{6}{*}{ 2. Síntomas neurofisiológicos } & 3. Debilidad de piernas & & .732 & & \\
\hline & 12. Manos temblorosas & & .708 & & \\
\hline & 13. Cuerpo tembloroso & & .703 & & \\
\hline & 6. Sensación de mareo & & .554 & & \\
\hline & 15. Dificultad para relajarse & & .545 & & \\
\hline & 19. Debilidad & & .494 & & \\
\hline \multirow{3}{*}{ 3. Síntomas respiratorios } & 15. Dificultad para respirar & & & .870 & \\
\hline & 11. Sensación de ahogo & & & .724 & \\
\hline & 7. Opresión en el pecho, o latidos acelerados & & & .624 & \\
\hline \multirow{6}{*}{ 4. Respuesta autonómica } & 2. Oleadas de calor (bochornos) & & & & .773 \\
\hline & 20. Ruborizarse, sonrojamiento & & & & .772 \\
\hline & 21. Sudoración (no debida a calor) & & & & .759 \\
\hline & \% Varianza explicada & 35.78 & 9.42 & 7.78 & 6.94 \\
\hline & \% Varianza acumulada & 35.78 & 45.21 & 52.99 & 59.93 \\
\hline & Coeficiente alfa de Cronbach & .858 & .755 & .783 & .757 \\
\hline
\end{tabular}

Método de extracción de análisis de componentes principales. Método de rotación: Varimax con normalización Kaiser. La rotación convergió en siete iteraciones.

La escala compuesta por los reactivos indicados mantuvo un índice de confiabilidad $\alpha$ de .89. En esta versión se obtuvo un puntaje mínimo de 0 y un máximo de 57 puntos; la puntuación media de la muestra fue de 10.25 (D.E. =9.26). Se encontraron diferencias estadísticamente significativas entre hombres y mujeres $(t=2.49, \mathrm{gl}=155, p<.05)$, de manera que la puntuación total media de las mujeres $(M=11.39$, D.E. $=9.37)$ fue mayor que la de los varones $(\mathrm{M}=7.38$, D.E. $=8.42)$.

En la Tabla 4 se muestra que los índices de correlación (r) de los factores con el total de la escala oscilaron entre muy alto y alto (de .87 a .65). Asimismo, el factor 3 (síntomas de respiración) obtuvo una correlación negativa moderada con el ACT $(r=-.55)$. 
Tabla 4. Índices de correlación entre los factores obtenidos y el total de las escalas de ansiedad, depresión y control del asma.

\begin{tabular}{|l|c|c|c|c|c|c|}
\hline \multicolumn{1}{|c|}{ Factor } & $\mathbf{1}$ & $\mathbf{2}$ & $\mathbf{3}$ & $\mathbf{4}$ & $\mathbf{5}$ & $\mathbf{6}$ \\
\hline 1. Síntomas subjetivos & & & & & & \\
\hline 2. Síntomas neurofisiológicos & $.56 * *$ & & & & & \\
\hline 3. Síntomas de respiratorios & $.54 * *$ & $.52 * *$ & & & & \\
\hline 4. Respuesta autonómica & $.45 * *$ & $.43 * *$ & $.34 * *$ & & & \\
\hline 5. BAI & $.87 * *$ & $.82 * *$ & $.74 * *$ & $.65 * *$ & & \\
\hline 6. BDI & $.87 * *$ & $.44 * *$ & $.34 * *$ & $.19 *$ & $.53 * *$ & \\
\hline 7. ACT & $-.35 * *$ & $-.27 * *$ & $-.55 * *$ & $-.16 *$ & $-.43 * *$ & $.45 * *$ \\
\hline
\end{tabular}

$* * p<.01$ (dos colas).

$* p<.05$ (dos colas).

Por otro lado, el reactivo 2 del ACT (falta de aire) correlacionó moderadamente con el total del factor 3 (síntomas de respiración) $(\mathrm{r}=.57, p<.01)$, así como con el reactivo de opresión en el pecho o latidos acelerados $(\mathrm{r}=.56, p<.01)$ y dificultad para respirar $(\mathrm{r}=.58)$ del BAI, aunque también obtuvo una correlación baja con sensación de ahogo $(\mathrm{r}=.35, p<.01)$.

Con los puntos de corte propuestos por Robles et al. (2001) para el instrumento de 21 ítems, se obtuvo una ansiedad mínima de $33.8 \%$, ansiedad leve de $36.9 \%$, ansiedad moderada de $22.9 \%$ y ansiedad severa de $6.4 \%$. Una vez realizado el procedimiento de relación de linealidad para la versión de 19 ítems, se sugiere un punto de corte de 0 a 4 puntos para ansiedad mínima, de 5 a 14 para ansiedad leve, de 15 a 27 para ansiedad moderada y de 28 puntos en adelante para ansiedad severa. Con estos puntos de corte se obtuvo una prevalencia de $31.2 \%$ de la muestra con ansiedad mínima, $40.8 \%$ con ansiedad leve, $21.7 \%$ con ansiedad moderada y $6.4 \%$ con ansiedad severa. En la Tabla 5 se muestran los valores percentiles para la muestra total de pacientes asmáticos, así como de hombres y mujeres.

Tabla 5. Puntuaciones percentiles del BAI para muestra total de adultos mexicanos con asma.

\begin{tabular}{|c|c|c|c|}
\hline Percentiles & $\begin{array}{c}\text { Total } \\
(\mathrm{n}=157)\end{array}$ & $\begin{array}{c}\text { Mujeres } \\
(\mathrm{n}=112)\end{array}$ & $\begin{array}{c}\text { Hombres } \\
(\mathrm{n}=45)\end{array}$ \\
\hline 5 & 0 & 0 & 0 \\
\hline 10 & 0 & 1 & 0 \\
\hline 20 & 2 & 3 & 1 \\
\hline 25 & 3 & 5 & 1 \\
\hline 30 & 4 & 6 & 2 \\
\hline 40 & 6 & 7 & 3 \\
\hline 50 & 8 & 9 & 4 \\
\hline 60 & 10 & 11 & 6 \\
\hline 70 & 13 & 15 & 9 \\
\hline 75 & 16 & 16 & 9 \\
\hline 80 & 17 & 17 & 13 \\
\hline 90 & 23 & 25 & 21 \\
\hline 95 & 32 & 34 & 29 \\
\hline 99 & 39 & 41 & - \\
\hline M & 10.24 & 11.39 & 7.38 \\
\hline D.E. & 9.26 & 9.37 & 8.42 \\
\hline
\end{tabular}




\section{DISCUSIÓN}

Los resultados obtenidos en el presente estudio apuntan a que la versión en español del BAI es una medida confiable y válida para ser utilizada con población asmática mexicana y muestra una consistencia interna adecuada de .89 , que es un valor ligeramente más bajo que el reportado en el estudio de Beck et al. (1988) $(\alpha=.92)$, superior al referido por Robles et al. (2001) en población mexicana $(\alpha=.83)$ e idéntico al obtenido en dos muestras de pacientes con enfermedades crónicas (Clark et al., 2016; Kagee et al., 2015).

Los resultados entre ansiedad y depresión obtenidos $(\mathrm{r}=.53, p<.01)$ señalan que estas dos sintomatologías están interrelacionadas moderadamente, pero el instrumento cuenta con la capacidad de discernir entre ambas entidades, lo que indica que la validez divergente es adecuada. La relación negativa moderada entre los síntomas de ansiedad y el control asmático $(\mathrm{r}=-.43, p<.01)$ confirma que los asmáticos con ansiedad suelen exhibir una mayor sintomatología asmática y viceversa, lo que concuerda con lo reportado por Lavoie et al. (2011) y Lomper et al. (2016).

Un primer análisis factorial arrojó la presencia de cuatro factores y un indicador compuesto por dos reactivos: 1 "entumecimiento" y 18 "indigestión o malestar estomacal". En virtud de los criterios que se emplearon para dejar una matriz factorial resultante (Reyes y García, 2008), se decidió prescindir de esos reactivos.

Una posible explicación de por qué estos síntomas no se agruparon en algún factor, es que podrían no ser considerados por los participantes como característicos de ansiedad, sino debidos a otras patologías médicas.

Por ende, se llevó a cabo un segundo análisis factorial en el que se conservaron cuatro factores: síntomas subjetivos, síntomas neurofisiológicos, síntomas de respiración y respuesta autonómica. Los factores obtenidos son muy similares a los obtenidos por Robles et al. (2001) en población mexicana y, en general, con lo reportado por otras investigaciones (p. ej., Bardoshi et al., 2016).

Es importante resaltar el factor denominado "síntomas de respiración", que hace referencia a los síntomas de ansiedad relacionados con el mecanismo de inhalación-exhalación. Dichos sínto- mas también son característicos de la enfermedad asmática (disnea, sensación de ahogo y opresión torácica). En una investigación de Borden, Peterson y Jackson (1991), realizada con población universitaria, y en otra de Lindsay y Skene (2007) con población con discapacidades intelectuales, se identificaron factores relacionados con síntomas de respiración y sensación de asfixia, respectivamente; sin embargo, en ninguno de esos estudios los tres reactivos que aquí se reportan se agruparon para formar un solo factor.

Tal factor, relacionado con los síntomas de respiración, se destaca aún más cuando se observa su fuerte relación con el ACT $(\mathrm{r}=-.55, p<.01)$, a diferencia de los demás factores; además, obtuvo una asociación moderada con el reactivo del ACT que específicamente cuestiona al paciente acerca de su sensación de falta de aire debida al asma $(\mathrm{r}=.57, p<.01)$.

De acuerdo con autores como Clark et al. (2016) y Phan et al. (2016), los pacientes con enfermedades médicas pueden interpretar los síntomas somáticos de la ansiedad de diferentes maneras dependiendo las características del padecimiento físico que les afecta, y la población asmática no parece ser la excepción.

En la práctica clínica, se podrían considerar los reactivos de este factor de síntomas respiratorios para contrastar el mismo con las pruebas pertinentes de función pulmonar. Si se indica un funcionamiento dentro de los criterios considerados como asma controlada, entonces se podría descartar que los síntomas sean una consecuencia de la enfermedad, y proceder entonces a tratar los síntomas mediante un tratamiento psicológico para la ansiedad.

Los hallazgos aquí presentados son consistentes con lo reportado en otras investigaciones en las que se observan diferencias significativas entre las puntuaciones de mujeres y hombres (Sanz, 2014). Autores como Bardoshi et al. (2016) han planteado que habría que cuestionar si los puntos de corte para indicar una ansiedad clínicamente relevante deben ser los mismos para estos dos grupos.

Por lo anterior, resulta útil el uso de tablas percentilares en virtud de que permiten acceder a una interpretación fácil de un grupo de referencia. Por ejemplo, según Robles et al. (2001), la norma para clasificar ansiedad severa es de 31 puntos y se ubica dentro del percentil 95. En los resultados 
aquí reportados, las mujeres obtuvieron resultados similares, con un puntaje de 34 puntos ubicado en el baremo 95; en el caso de los varones, una puntuación cruda de 29 puntos es indicativa de que el participante se ubica por encima de $95 \%$ de su grupo, lo que podría considerarse una ansiedad más severa.

De este modo, conocer la puntuación del paciente en términos de su percentil y no sólo de un determinado punto de corte ayuda a entender la ansiedad no únicamente como una categoría, sino también a conocer cómo se ubica un individuo res- pecto a su grupo particular de referencia, lo que ayudaría al profesional de la salud a llevar a cabo una evaluación más certera y a tomar mejores decisiones clínicas respecto a su manejo y tratamiento.

Es recomendable que futuros estudios se auxilien de pruebas de función pulmonar para realizar la contrastación entre la disnea reportada por el paciente y la obstrucción real del flujo aéreo. De igual manera, sería de provecho emplear una medida de comparación, como una entrevista clínica diagnóstica, que haga posible establecer la presencia de trastornos de ansiedad.

\section{REFERENCIAS}

Bardhoshi, G., Duncan, K. y Erford, B. (2016). Psychometric meta-analysis of the English version of the Beck Anxiety Inventory. Journal of Counseling \& Development, 94(3), 356-373. doi: 10.1002/jcad.12090.

Beck, A.T., Epstein, N., Brown, G. y Steer, R.A. (1988). An inventory for measuring clinical anxiety: Psychometric properties. Journal of Consulting and Clinical Psychology, 56, 893-897. doi: 10.1037/0022-006X.56.6.893.

Beck, A.T. y Steer, R.A. (1991). Relationship between the Beck Anxiety Inventory and the Hamilton Anxiety Rating Scale with anxious outpatients. Journal of Anxiety Disorders, 5, 213-223. doi: 10.1016/0887-6185(91)90002-B.

Beck, A.T., Steer, R.A. y Brown, G.K. (1996). BDI-II. Beck Depression Inventory-Second Edition manual. San Antonio, TX: The Psychological Corporation.

Borden, J., Peterson, D. y Jackson, E. (1991). The Beck Anxiety Inventory in nonclinical samples: Initial psychometric properties. Journal of Psychopathology and Behavioral Assessment, 13(4), 345-356.

Braket, A., Zawada, K., Beil-Gawetcytz, J., Beil, S., Sozanska, E., Krysta, K. y Pierzchala, W. (2015). Depressiveness, symptoms of anxiety and cognitive dysfunctions in patients with asthma and chronic obstructive pulmonary disease (COPD): possible associations with inflammation markers: a pilot study. Journal of Neural Transmission, 122, 83-91. doi: 10.1007/s00702014-1171-9.

Brumpton, B.M. Leivseth, L., Romunddstad, P., Langhammer, A., Chen, Y. y Camargo, A. (2013). The joint association of anxiety, depression and obesity with incident asthma in adults: the HUNT Study. International Journal of Epidemiology, 42(5), 1455-1463. doi: 10.1093/ije/dyt151.

Caballero S., N., Fernández V., M., Salas H., J., Chapela M., R. y Sánchez-Sosa, J.J. (2009). Efectos de una intervención psicológica cognitivo-conductual sobre la adhesión terapéutica y la ansiedad en pacientes asmáticos. Revista Española de Asma, $1,6-15$.

Carretero D., H. y Pérez, C. (2005). Normas para el desarrollo y revisión de estudios instrumentales. International Journal of Clinical and Health Psychology, 5, 521-551.

Ciprandi, G., Schiavetti, I., Rindone, E. y Ricciardolo, F. (2015). The impact of anxiety and depression on outpatients with asthma. Annals of Allergy and Asthma Immunology, 115(5), 408-414. doi: 10.1016/j.anai.2015.08.0071-7.

Clark, J., Marszalek, J., Bennett, K., Harry, K., Howarter, A., Eways, K. y Reed, K. (2016). Comparison of factor structure models for the Beck Anxiety Inventory among cardiac rehabilitation patients. Journal of Psychosomatic Research, 89, 91-97. doi: 10.1016/j.jpsychores.2016.08.007.

Coban, H. y Aydemir, Y. (2014). The relationship between allergy and asthma control, quality of life, and emotional status in patients with asthma: a cross-sectional study. Allergy, Asthma and Clinical Immunology, 10, 78-90. doi: 10.1186/s13223014-0067-4.

Contreras, S., Fernandez, S., Malcarne, V.L., Ingram, R.E. y Vaccarino, V.R. (2004). Reliability and validity of the Beck Depression and Anxiety inventories in Caucasian Americans and Latinos. Hispanic Journal of Behavioral Sciences, 26, 446-462. doi: 10.1177/0739986304269164.

Cooper, C., Parry, G., Saul, C., Morice, A., Hutchcroft, B., Moore, J. y Esmonde, L. (2007). Anxiety and panic fear in adults with asthma: prevalence in primary care. BMC Family Practice, 8(1), 62. doi: 10.1186/1471-2296-8-62.

Correia de S., J., Pina, A., Cruz, A., Quelhas, A., Almada L., F., Cabrita, J. y Yaphe, J. (2013). Asthma control, quality of life, and the role of patient enablement: a cross-sectional observational study. Primary Care Respiratory Journal, 22(2), $181-187$. doi: $10.4104 /$ pcrj.2013.00037. 
Durham, T.L. (2010). Reliability and validity of the Beck Anxiety Inventory for deaf college students. Dissertation Abstracts International: Section B. Sciences and Engineering, 70(8), 51-57.

Global Initiative for Asthma (GINA) (2015). 2015 GINA Report. Disponible en http://www.ginasthma.org/.

Goodwin, R.D., Jacobi, F. y Thefeld, W. (2003). Mental disorders and asthma in the community. Archives of General Psychiatry, 60, 1125-30. doi: 10.1001/archpsyc.60.11.1125.

Hamilton, M. (1967). Development of a rating scale for primary depressive illness. British Journal of Social Clinical Psychology, 6, 278-296.

Hamilton, M.C. (1969). Diagnosis and rating of anxiety. British Journal of Psychiatry, 3, 76-79.

Jurado, S., Villegas, M., Méndez, L., Rodríguez, F., Loperena, V. y Varela, R. (1998). La estandarización del Inventario de Depresión de Beck para los residentes de la Ciudad de México. Salud Mental, 21(3), 26-31.

Kabacoff, R.I., Segal, D.L., Hersen, M. y Van Hasselt, V.B. (1997). Psychometric properties and diagnostic utility of the Beck Anxiety Inventory and the State-Trait Anxiety Inventory with older adult psychiatric outpatients. Journal of Anxiety Disorders, 11, 33-47. doi: 10.1016/S0887-6185(96)00033-3.

Kagee, A., Coetzee, B., Saal, W. y Nel, A. (2015). Using the Beck Anxiety Inventory Among South Africans Living With HIV: Exploratory and Higher Order Factor Analyses. Measurement and Evaluation in Counseling and Development, 48(3), 204213. doi: $10.1177 / 0748175615578734$.

Kumar, G., Steer, R.A. y Beck, A.T. (1993). Factor structure of the Beck Anxiety Inventory with adolescent psychiatry inpatients. Anxiety, Stress and Coping, 6, 125-131. doi: 10.1080/10615809308248374.

Larenas L., D., Salas H., J., Vázquez G., J.C., Ortiz A., I., Fernández V., M. y Del Río N., B.E. (2017). Guía mexicana del asma. Revista Alergia México, 64(1), 11-128.

Lavoie, K., Bodreau, M., Plourde, A., Campbell, T. y Bacon, S. (2011). Association between generalized anxiety disorder and asthma morbidity. Psychosomatic Medicine, 73, 504-513. doi: 10.1097/PSY.0b013e318222e9fc.

Li, L.H., He, X. L., Liang, B.M., Zhang, P.H., Wang, Y. y Wang, G. (2015). Anxiety but not depression symptoms are associated with greater percived dyspnea in asthma during bronchoconstriction. Allergy and Asthma Proceedings, 36(6), 447-457. doi: 10.2500/aap.2015.36.3897.

Lindsay, W.R. y Skene, D.D. (2007). The Beck Depression Inventory II and the Beck Anxiety Inventory in people with intellectual disabilities: Factor analyses and group data. Journal of Applied Research in Intellectual Disabilities, 20, 401-408. doi: 10.1111/j.1468-3148.2007. 00380.x.

Liu, S., Wu, R., Li, L., Li, G., Zhang, X., Guo, Y., Wang, Y. y Li, H. (2014). The prevalence of anxiety and depression in Chinese asthma patients. PLOS ONE, 9(7), e103014. doi: 10.1371/journal.pone.0103014.

Lomper, K., Chudiak, A., Uchmanowicz, I., Rosinczuk, J. y Jankowska-Polanska, B. (2016). Effects of depression and anxiety on asthma-related quality of life. Pneumonologia i Alergologia Polska, 84(4), 212-221. doi: 10.5603/PiAP.2016.0026.

Lovibond, P.F. y Lovibond, S.H. (1995). The structure of negative emotional states: Comparison of the Depression Anxiety Stress Scales (DASS) with the Beck Depression and Anxiety Inventories. Behavior Research and Therapy, 33, 335-343. doi: 10.1016/0005-7967(94)00075.

Lugo G., I., Reynoso E., L. y Fernández V., M. (2014). Percepción de enfermedad, depresión, ansiedad y control del asma: una primera aproximación. Neumología y Cirugía de Tórax, 73(2), 114-121.

Morin, C.M., Landreville, P., Colecchi, C., McDonald, K., Stone, J. y Ling, W. (1999). The Beck Anxiety Inventory: Psychometric properties with older adults. Journal of Clinical Geropsychology, 5, 19-29. doi: 10.1023/A:1022986728576.

Nathan, R.A., Sorkness, C.A., Kosinski M, Schatz, M., Li, T., Marcus, P., Murray, J. y Pendergraft, T. (2004). Development of the Asthma Control Test: A survey for assessing asthma control. Journal of Allergy and Clinical Immunology, 113, 59-65.

Osman, A., Barrios, F.X., Aukes, D., Osman, J.R. y Markway, K. (1993). The Beck Anxiety Inventory: Psychometric properties in a community population. Journal of Psychopathology and Behavioral Assessment, 15, 287-297. doi: 10.1007/BF00965034.

Osman, A., Hoffman, J., Barrios, F.X., Kopper, B.A., Breitenstein, J.L. y Hahn, S.K. (2002). Factor structure, reliability, and validity of the Beck Anxiety Inventory in adolescent psychiatric inpatients. Journal of Clinical Psychology, 58, 443-456. doi: $10.1002 /$ jclp. 1154 .

Peterson, D.R. (1995). Structural analysis of the Beck Anxiety Inventory across a clinical and student population. Dissertation Abstracts International: Section B. Sciences and Engineering, 56(3), 1741.

Phan, T., Carter, O., Adams, C., Waterer, G., Ching, L. P. y Strobel, N. (2016). Discriminant validity of the Hospital Anxiety and Depression Scale, Beck Depression Inventory (II) and Beck Anxiety Inventory to confirmed clinical diagnosis of depression and anxiety in patients with chronic obstructive pulmonary disease. Chronic Respiratory Disease, 13(3), 220-228. doi: $10.1177 / 1479972316634604$.

Reyes L., I. y García B., L.F. (2008). Procedimiento de validación psicométrica culturalmente relevante: Un ejemplo. En S. Rivera-Aragón, R. Díaz-Loving y R. Sánchez-Aragón, R. (Eds.) La Psicología Social en México, 12 (pp. 625-636). México: Asociación Mexicana de Psicología Social. 
Ritz, T., Meuret, A.E., Trueba, A., Fritzche, A. y von Leupoldt, A. (2013). Psychosocial factors and behavioral medicine interventions in asthma. Journal of Consulting and Clinical Psychology, 81(2), 231-250. doi: 10.1037/a0030187.

Robles, R., Varela, R., Jurado, S. y Páez, F. (2001). Versión mexicana del Inventario de Ansiedad de Beck: propiedades psicométricas. Revista Mexicana de Psicología, 18(2), 211-218.

Sanford, S.D., Bush, A.J., Stone, K.C., Lichstein, K.L. y Aguillard, N. (2008). Psychometric evaluation of the Beck Anxiety Inventory: A sample with sleep-disordered breathing. Behavioral Sleep Medicine, 6, 193-205. doi: 10.1080/15402000802162596.

Sanz, J. (2014). Recomendaciones para la utilización de la adaptación española del Inventario de Ansiedad de Beck (BAI) en la práctica clínica. Clínica y Salud, 25, 39-48.

Steer, R.A. (2009). Amount of general factor saturation in the Beck Anxiety Inventory: Responses of outpatients with anxiety disorders. Journal of Psychopathology \& Behavioral Assessment, 31, 112-118. doi: 10.1007/s10862-008-9098-9.

Steer, R.A., Kumar, G. y Beck, A.T. (1993). Self-reported suicidal ideation in adolescent psychiatric inpatients. Journal of Consulting and Clinical Psychology, 61, 1096-1099. doi: 10.1037/0022-006X.61.6.1096.

Steer, R.A., Kumar, G., Ranieri, W.F. y Beck, A.T. (1995). Use of the Beck Anxiety Inventory with adolescent psychiatric outpatients. Psychological Reports, 76, 459-465. doi: 10.2466/pr0.1995.76.2.459.

Steer, R.A., Rissmiller, D.J., Ranieri, W.F. y Beck, A.T. (1993). Structure of the computer-assisted Beck Anxiety Inventory with psychiatric inpatients. Journal of Personality Assessment, 60, 532-542. doi: 10.1207/s15327752jpa6003_10.

Tany, R.F. y Saha, A.K. (2017). A study on stress and anxiety in relation to asthma. Journal of Psychosocial Research, 12, 117126. doi: 10.1186/s40733-015-0007-1. 